\title{
ESTUDIO SOBRE EL POSICIONAMIENTO Y LA VALIDACIÓN DEL ARTISTA EN EL ÁMBITO CULTURAL DE NUEVO LEÓN, MÉXICO
}

\section{STUDY ON POSITIONING AND VALIDATION OF ARTIST IN THE CULTURAL DOMAIN OF NUEVO LEÓN, MÉXICO}

\section{Oliva Abarca, Jesus ${ }^{1}$}

\section{Resumen}

En este trabajo se examinan las condiciones de posicionamiento y las estrategias que validan las actividades del artista como actor cultural en el campo artístico de Nuevo León, México. El diseño de la investigación fue descriptivo, y la metodología empleada fue cualitativa, recurriendo a la entrevista semiestructurada como estrategia de investigación, con el propósito de obtener información relevante desde la perspectiva de los actores involucrados en el fenómeno a examinar; el tipo de muestra se basó en las opiniones y valoraciones de once expertos y la información recopilada se analizó por medio de la estructuración de los datos obtenidos en temas descriptivos. De entre los resultados de investigación obtenidos se pueden destacar, primero, el que una de las condiciones de posicionamiento del artista radica en su capacidad para desempeñar diferentes roles, tales como creador, promotor, o docente, y, segundo, que su validación como actor cultural depende de su participación en contextos institucionales, así como de circunstancias externas al campo artístico.

Palabras clave: actor cultural, campo artístico, campo de producción cultural, sociología del arte, sociología del reconocimiento

\begin{abstract}
In this paper, it will be examined the conditions of positioning and the strategies by which an individual is accredited as a cultural agent. This research focuses on the analysis of a specific cultural actor, the artist, and its incorporation and development in the artistic field of the Mexican state of Nuevo León. The research design chosen was descriptive, while qualitative methodology was the most accurate approach for this subject. To obtain pertinent data from the involved actors,
\end{abstract}

\footnotetext{
${ }^{1}$ Universidad Autónoma de Nuevo León, México. Correo: JESUS.OLIVAABR@uanl.edu.mx
} 
the semistructured interview was the research strategy chosen. The sample was constructed on the participation of eleven experts. The information obtained was analyzed by structuring the data collected in descriptive themes. From the results, it can be highlighted that, in order to gain a position inside the artistic field, an artist must be capable of fulfilling different roles, as creator, as a promoter, or as a professor. Also, his/her validation as a cultural agent will depend on his/her involvement in institutional contexts.

Keywords: artistic field, cultural agent, field of cultural production, sociology of art, sociology of recognition

\section{Introducción}

El arte se constituye por prácticas, normas y códigos regulados que le otorgan el estatus de sistema, es decir, como una estructura compleja en la que diversos elementos interactúan entre sí, estableciendo relaciones dinámicas. Precisamente el análisis de estas interacciones se ha convertido en el interés central de muchos estudiosos que ya no conciben el campo artístico como un reino separado de los otros dominios de las actividades humanas. Si bien, desde una óptica teórica, se reconoce la autonomía y la orientación autotélica del mundo del arte, de igual manera se insiste en la premisa de que los sistemas artísticos están estrechamente relacionados con ámbitos tales como la economía, la política y todas las demás esferas que constituyen las sociedades humanas. Así pues, los estudios sobre el arte ya no adoptan la concepción inmanentista tradicional, según la cual atributos como la belleza o la armonía eran rasgos inherentes a la obra de arte, y los artistas eran considerados como individuos excepcionalmente creativos. Hoy en día, los enfoques sociológicos del arte adquieren una mayor aceptación, dado que el principal interés de investigación lo constituyen los procesos de producción, distribución y recepción de las expresiones artísticas.

Aunado a lo anterior, son cada vez más los organismos e instituciones, así

como los teóricos y analistas, que se suman a la idea de que la cultura es la 
expresión más fidedigna del progreso y del desarrollo humano. Precisamente la UNESCO promueve una caracterización de la cultura como potencia transformadora con potencial para impulsar las economías locales y el bienestar social de todos los países (PNUD, 2014). Esta línea de pensamiento se apoya en el crecimiento del sector productivo de las industrias culturales y creativas, pertenecientes a los paradigmas económicos denominados como economía cultural y economía creativa respectivamente (O'Connor, 2010). Dado que muchos gobiernos han reconocido las actividades culturales y creativas como las bases de una nueva economía (PNUD, 2014), los discursos y concepciones respecto a las manifestaciones artísticas han cambiado, y por lo tanto, los artistas también han adaptado sus prácticas a las exigencias de los nuevos sistemas sociales, para así asegurar, o mantener, su posición en el campo artístico (Stallabras, 2004).

El propósito de esta investigación fue el de analizar las estrategias empleadas y los valores a realizar por los individuos cuya aspiración es posicionarse en el ámbito de las artes, y ser acreditados o reconocidos como agentes culturales, ya sea como artistas, promotores o gestores culturales, galeristas o como docentes orientados a la educación artística. Este estudio estuvo limitado a Nuevo León, estado localizado al noreste de México, concretamente a los municipios de Monterrey y San Pedro Garza García, y se orientó al sector cultural de las artes visuales. Junto con la ciudad de México y Guadalajara, Nuevo León es, tanto en términos culturales como económicos, una de las zonas geográficas más sobresalientes del país. Un objetivo secundario que debe ser mencionado es el de obtener un panorama del funcionamiento interno del campo artístico a través de las perspectivas y opiniones de sus propios actores.

\section{Contexto del campo artístico neoleonés}

La vida artística en el estado mexicano de Nuevo León ha estado ligada a la actividad de personas acaudaladas, cuyo interés en la cultura y las artes ha derivado en la creación de galerías, museos, centros culturales e instituciones educativas. Aunque ha habido iniciativas gubernamentales cuyo objetivo es promover la 
producción cultural local, el presupuesto público se ha destinado con mayor frecuencia a la industria manufacturera. El alto grado de industrialización del estado ha dado por resultado que el desarrollo artístico y cultural ocupe un segundo plano en las políticas públicas. No obstante, se debe destacar el trabajo del Consejo para la Cultura y las Artes de Nuevo León (CONARTE) y de la Universidad Autónoma de Nuevo León (UANL), ambas instituciones públicas consolidadas cuya prioridad es el desarrollo de nuevos talentos artísticos y la promoción y difusión de la cultura neoleonesa.

Además de los espacios culturales y educativos públicos, el ámbito artístico local incluye iniciativas privadas, fundadas y administradas por personas que cuentan con el capital necesario, e iniciativas culturales independientes, establecidas por jóvenes emprendedores con pocos o nulos recursos. La escena artística neoleonesa se conforma, entonces, por actores adscritos a diferentes ámbitos de actuación: por una parte, aquellos pertenecientes a instituciones públicas, por otra, los posicionados desde la iniciativa privada empresarial, y finalmente, quienes, independientemente de la orientación que posean, intervienen en la esfera artística de manera independiente, es decir, sin adscripción a organismos públicos o empresas privadas. Las relaciones, intercambios e interacciones simbólicas (Bourdieu, 2002) que estos tres tipos de actores establecen entre sí permite esbozar los modos de operación del campo artístico neoleonés. Para dar una visión más completa del campo artístico de Nuevo León, es pertinente realizar una somera revisión a sus instituciones más relevantes.

De la década de 1940 a 1960, fue mayor y más significativa la implicación de los empresarios locales en la vida cultural neoleonesa (Amores, 2007). Como resultado, se fundaron dos organizaciones que hasta el día de hoy perduran; la primera, la Sociedad Artística Tecnológico (SAT), se estableció con el apoyo del Instituto Tecnológico de Estudios Superiores de Monterrey (ITESM), y de todos sus socios comerciales y de sus benefactores, y cuyo objetivo es el de la promoción de eventos artísticos a un público amplio. La segunda, Arte A.C., tenía por propósito original ofrecer cursos de dibujo y de pintura a toda persona interesada en el arte, y 
realizar exposiciones gratuitas de talentos artísticos locales. Hoy en día, Arte A.C. es una institución cultural y educativa consolidada en la región.

Otro espacio cultural importante es el Centro Cultural Alfa, creado en los años 70 , con el objetivo de atraer inversionistas y socios de negocios para los empresarios que lo fundaron; no obstante, cambió rápidamente su orientación comercial a la cultural, con exhibiciones de avances científicos y tecnológicos y funciones cinematográficas (Amores, 2007). Las acciones de los empresarios locales parecieron incentivar la participación del gobierno en la cultura, pues, en ese mismo período se creó la Dirección Estatal de Cultura, organismo financiado con presupuesto estatal cuyo objetivo era el de impulsar la vida artística y cultural del Estado (Rubio-Elosúa, 2000); cabe señalar que ésta fue predecesora y antecedente de CONARTE, la institución cultural más importante del estado.

Es pertinente mencionar que existen ejemplos de una alianza entre el gobierno y los intereses de los empresarios en la vida cultural del Estado (NivónBolán y Villalobos-Audifred, 2006), los casos destacados de dicha colaboración son el Museo de Arte Contemporáneo (MARCO) y el Parque Fundidora. El primero, MARCO, mantiene numerosos convenios centrados en la educación artística con escuelas de nivel básico y con la Universidad Autónoma de Nuevo León; el Parque Fundidora, por su parte, alberga centros culturales públicos, tales como la Cineteca, el Centro de las Artes y la Escuela Adolfo Prieto. El panorama descrito hasta aquí evidencia la estrecha relación y las constantes interacciones entre los actores culturales adscritos a instituciones públicas y aquellos vinculados con centros culturales privados.

Bajo estas circunstancias, el campo producción cultural local pareciera estar dividido en dos facciones con ideologías aparentemente opuestas. Por un lado, el grupo integrado por trabajadores culturales pertenecientes a organismos gubernamentales y artistas independientes, cuyas actividades están enfocadas a la transformación social, al cumplimiento de valores culturales, y a la experimentación artística. Por otro lado, el bando formado por personas pudientes, quienes se orientan a la creación artística y a la comercialización de bienes y servicios 
culturales (Stallabras, 2004). Dados los frecuentes recortes presupuestales a la cultura y las artes, ambas partes, pese a sus diferencias ideológicas, han tenido que buscar múltiples alternativas para sostener sus iniciativas. Ante esta situación, la autogestión se ha convertido en una competencia valiosa que debe adquirir toda persona que aspire a involucrarse en la escena cultural neoleonesa.

\section{El campo artístico como sistema social}

La investigación en torno a las artes se ha apoyado en múltiples perspectivas: desde la filosofía hasta la sociología y la psicología, e incluso la economía, los estudios sobre el arte han recurrido a estas disciplinas para intentar explicar los complejos procesos por medio de los cuales se valida el arte, cómo un individuo adquiere el estatus de artista y de qué manera un tipo de objetos son legitimados como bienes artísticos y/o culturales. En el caso específico del presente estudio, el enfoque sociológico fue el más adecuado, pues se requirió abordar el campo artístico como un sistema de jerarquías y estratificaciones (Alexander, 2000) en el que sus actores se desplazan e interactúan de diversas maneras. Además, la teoría del campo de Bourdieu es un referente teórico necesario para el análisis de los elementos, agentes y procesos que constituyen el espacio de las expresiones culturales; precisamente su noción de campo de la producción cultural permite una comprensión del dominio artístico o literario como campos en los cuales las relaciones e interacciones entre los actores involucrados dependerán de las posiciones que ocupen o tomen y de las formas en que legitimen sus actividades (Bourdieu, 2002).

La sociología del fenómeno artístico de Bourdieu constituye una respuesta, y una alternativa, a las teorías subjetivistas o inmanentistas sobre el arte; el concepto de campo formulado por Bourdieu concibe a las obras de arte no como objetos cuyo valor reside en propiedades metafísicas, sino como bienes que son validados por los intercambios y las relaciones que establecen entre sí artistas, intermediarios y público, así como del grado de competencia artística que cada agente posea, esto es, del dominio del código, o códigos, que rigen al sistema artístico (p. 72). En lo 
que respecta específicamente a los actores de dicho sistema, o campo, Bourdieu señala que

las relaciones entre cada uno de los agentes del sistema y los agentes o las instituciones total o parcialmente externas al sistema, siempre están mediatizadas por las relaciones que se establecen en el seno mismo del sistema, es decir, en el interior del campo intelectual, y la competencia por la legitimidad cultural, cuya apuesta y, al menos en apariencia, cuyo árbitro, es el público, nunca se identifica completamente con la competencia por el éxito en el mercado (p. 15).

Tales relaciones por las cuales un individuo adquiere legitimidad cultural se pueden clasificar en tres tipos. La primera consiste en el reconocimiento que dan al artista otros agentes culturales, ya sea como colaboradores o incluso como competidores. La segunda es la validación otorgada por las élites culturales y las entidades culturales públicas y privadas. La tercera y última fase se refiere a las preferencias populares o masivas (p. 33-34). Estos vínculos se corresponden precisamente con los procesos de posicionamiento, de validación y de consagración del agente cultural como artista. En Las reglas del arte (1992), Bourdieu profundiza en la dialéctica del campo artístico y literario a través de un recorrido histórico que expone las condiciones económicas y políticas que contribuyen a la constitución del dominio artístico como ámbito que tiende a la autonomía, así como las operaciones predominantes en el campo artístico y las formas de interacción de los actores involucrados. Es necesario agregar que la teoría del campo cultural desarrollada por Bourdieu constituye uno de los referentes teóricos más importantes de esta investigación, además de que su aplicación no se restringe, en este estudio, sólo a los artistas, sino que contempla a los agentes culturales directamente imbricados en el campo artístico neoleonés, tales como galeristas, promotores y docentes.

La teoría del campo cultural de Bourdieu es la más socorrida en el estudio sociológico del arte, no obstante, existen otros planteamientos teóricos igualmente 
pertinentes para examinar el funcionamiento social y la organización del sistema artístico, así como las actividades de sus actores. Precisamente una orientación teórica similar es la elaborada por Niklas Luhmann, cuyo punto de partida consiste en abordar el mundo del arte como un sistema social de objetos artificiales producidos a partir de la creatividad humana, es decir, obras de arte, cuya peculiaridad es establecer una realidad autotélica (Luhmann, 2005, p. 237). Desde este punto de vista, la función del arte es introducir una relación entre una realidad "real" y una ficticia para hacer patente los fallos y aciertos de las estructuras sociales, ya sea

imitando la realidad al hacer ver lo que no ofrece de por sí -por ejemplo sus formas esenciales, sus ideas, su perfección divina; criticándola en lo que no admite de buena gana: sus insuficiencias, su dominio de 'clases', su orientación exclusivamente comercial, o afirmándola gracias a la representación que se logra (y que se logra tan bien), que se vuelve un placer producir la obra de arte y contemplarla (p. 238).

Desde la perspectiva de Luhmann (2005), la propiedad mimética del arte no se orienta al objeto o sujeto imitado como tal, sino a la red de relaciones que éste mantiene con su entorno, con otros objetos y sujetos; en otras palabras, el arte funciona más como un sistema de representaciones sociales que como una forma de comprensión ontológica. A diferencia de otros teóricos del arte, Luhmann (2005) enfatiza que la conexión que el arte, como dispositivo cultural mimético, establece con la realidad es, posiblemente, su principal función: "se podría decir también que la función del arte es hacer que el mundo aparezca dentro del mundo" (p. 249).

Por su parte, el planteamiento de Dickie (2001) respecto de la dimensión social del arte se sustenta en la premisa de que éste es una práctica institucional que ha de producir artefactos estéticos, los cuales serán validados como obras de arte o serán rechazados y excluidos del ámbito artístico. El filósofo señala que cualquier teoría del arte implica evaluar la validez de las prácticas que se denominan 
como artísticas (p. 98), por consiguiente, la obra de arte debe ser mostrada al mundo en general, y de manera particular, en un espacio donde pueda ser evaluada por cualquier persona involucrada en el mundo del arte (p. 107). Existe cierta similitud entre la tesis de Dickie y las ideas sostenidas por Benjamin en su célebre ensayo La obra de arte en la época de su reproductibilidad técnica, al sostener que toda obra de arte posee dos valores, el de culto y el de exposición, y que es precisamente en función de éste último por medio del cual el arte se torna en un objeto, o grupo de objetos, accesibles al público generalizado (Benjamin, 2003).

Retornando a la noción de campo de producción cultural, Bourdieu afirmaba que éste está constituido por factores tanto internos como externos. Las teorías comentadas anteriormente confirman precisamente que la ponderación de la obra, la función del arte y el papel del artista como agente cultural están en función de los valores y prácticas de evaluación que se desarrollan dentro y fuera del campo. Cada proceso que atañe a la constitución del campo artístico cambia de acuerdo a los avances tecnológicos, a los cambios sociales y culturales que reorganizan nuestra vida cotidiana. En la actualidad, el ámbito artístico se reconfigura con mayor rapidez debido a la integración económica global conocida como globalización (Hartley et al., 2013) y a los nuevos modelos de producción y consumo cultural que son las industrias culturales (Throsby, 2008) o industrias creativas (Davies y Sigthorsson, 2013).

En este contexto, los artistas se convirtieron en una parte sustancial de lo que Florida (2012) ha llamado la clase creativa, una nueva clase compuesta por trabajadores intelectuales, personas que crean nuevas ideas, tecnología innovadora y contenido creativo. Estos trabajadores creativos asumen y promueven los valores generalmente atribuidos a los artistas, como la individualidad, la autoexpresión, la diversidad y la apertura (p. 56-58). Además de las cualidades mencionadas, los principales atributos que los agentes culturales deben poseer en el marco de la economía global son la creatividad y la tendencia a la innovación (Howkins, 2013), valores que caracterizan a la economía creativa, sector económico constituido por 
las expresiones artísticas y culturales, el diseño, los medios de comunicación y el desarrollo y la investigación (p. 151).

Diversos estudiosos del fenómeno sostienen que las industrias culturales y creativas implican que la figura del artista se reafirma no sólo como un agente cultural sino también como un actor clave económico (Florida, 2012; Howkins, 2013). Para otros investigadores, antes que una revaloración del papel social del artista, se trata más bien de la estetización de la economía (O'Brien, 2014), lo que significa que el campo de producción cultural adopta un modelo organizacional semejante al del mundo empresarial (Davies y Sigthorsson, 2013). En este paradigma de la cultura y la creatividad como recursos para el desarrollo económico, los valores culturales y artísticos forman parte de un discurso universal en el que las obras y las prácticas se ponderan de acuerdo a criterios económicos (Guillory, 1994, p. 323). Los antecedentes de esta articulación entre valores estéticos, artísticos y culturales y estimaciones económicas datan, como señala Moulin (2012), de las décadas de los 70 y 80 , época en la que los mercados locales comienzan una paulatina apertura internacional a la vez que los empresarios-galeristas y marchands fortalecen sus actividades a través de estrategias de control de la ofertademanda artística (p. 38-39).

A pesar de que la dimensión económica de los bienes culturales es mayormente reconocida hoy en día, ello no resuelve el problema un problema de la valoración de los productos y procesos del campo cultural (Towse, 2010). La dificultad para definir y delimitar el valor cultural radica en que éste comprende e involucra cualidades estéticas, propiedades espirituales, factores sociales e históricos y rasgos simbólicos (Throsby, 2008). Además, el valor cultural no se concibe necesariamente como una propiedad inherente y atemporal perteneciente a un tipo especial de objetos y prácticas; antes bien, puede entenderse como un proceso en el que las evaluaciones sobre la validez de tal o cual objeto depende de las negociaciones llevadas a cabo entre los agentes involucrados (p. 45). De ello se puede inferir que existe una conexión recíproca entre la valoración de las prácticas y productos culturales y el posicionamiento de los agentes culturales al interior del 
campo de la producción cultural, ya que las operaciones de evaluación influyen y son influenciadas al mismo tiempo por las posiciones de los actores en el campo.

El valor cultural, entonces, pudiera definirse como una operación de intercambios ya sea entre significados, expectativas e interpretaciones o entre cualidades culturales y posibilidades económicas (Groys, 2014). Así pues, la estructura y el funcionamiento de los sistemas artísticos, así como sus valores y prácticas, están influenciados no sólo por factores económicos, sino también políticos y sociales. A propósito de este tema, Maanen (2009) examina las bases operacionales del campo artístico precisamente desde la óptica social, económica y política. Desde el punto de vista de Maanen, la organización de cualquier sistema artístico depende de condiciones económicas específicas, que son los tipos de financiamiento para proyectos artísticos e iniciativas culturales (Maanen, 2009). Además, hay circunstancias políticas que se refieren al grado de libertad artística y cultural y al tipo de valores que promueven un régimen político (p. 228). Aunado a todo ello, cada expresión artística o cultural se desarrolla en un contexto de distribución en el que interactúan entidades y agentes directamente involucrados con la cultura y las artes, tales como artistas, promotores y gestores culturales, curadores, museos, galerías, universidades, etc. (p. 244).

Los planteamientos anteriormente comentados conducen a un enfoque según el cual el arte se concibe como un universo social en sí, constituido por actores, prácticas, instituciones, reglas, objetos y conceptos propios que se desarrollan de manera conjunta (Heinich, 2002). Si bien, como indican Heinich (2002) y García-Canclini (2014), y otros, la sociología del arte comienza explorando el lugar del arte en la sociedad, el interés de las investigaciones se orienta posteriormente a las interacciones entre arte y sociedad, hasta postular la idea de que el "arte es, entre otras, una forma de actividad social que posee sus características propias" (Heinich, 2002, p. 43), o, de acuerdo a García-Canclini (2014), es el resultado de las relaciones sociales entre artistas, intermediarios y públicos (p. 37). Un aporte valioso para este estudio, y que complemente el enfoque sociológico aquí expuesto, es el de Peist (2011), quien explora los mecanismos de 
reconocimiento en el campo artístico a partir de la interacción entre artistas, coleccionistas, museos y críticos. De acuerdo a esta autora, el reconocimiento se produce en dos momentos, el primero consistente en la validación del artista por parte de sus pares, de la crítica, del mercado y del público (p. 316), y el segundo consiste en la institucionalización del artista, o lo que es lo mismo, la aceptación e incorporación de éste por parte de las instituciones (p. 323).

Si bien el marco teórico de este estudio se basó principalmente en la teoría del campo artístico de Bourdieu, es conveniente señalar que el análisis de Maanen sobre la organización y el funcionamiento social del ámbito artístico fue también un enfoque fundamental para esta investigación. La tesis de Bourdieu acerca del campo cultural como un espacio de relaciones y posiciones, así como sus ideas de los principios de jerarquización, fueron las bases teóricas principales para analizar el posicionamiento de los actores culturales en función de criterios autónomos y heterónomos (Bourdieu, 2002). De Maanen se retoma su examen de los factores que intervienen en los procesos de producción, distribución y recepción artística y/o cultural (Maanen, 2009), como apoyo para definir los procesos por los cuales un individuo determinado ingresa, se posiciona y es acreditado como un agente cultural.

\section{Método}

Para este estudio, se optó por un diseño de investigación descriptivo, para con ello identificar patrones que operan bajo ciertas circunstancias (Walliman, 2011); en este caso en específico, determinar los aspectos que intervienen en la validación y en el posicionamiento de los individuos involucrados como creadores en el campo artístico neoleonés. Dado que el enfoque cualitativo tiene por objetivo investigar los valores, actitudes y comportamientos que caracterizan situaciones sociales y formas organizacionales particulares (Álvarez-Gayou, 2012), es el enfoque más adecuado para el propósito de esta investigación, consistente en, como se ha señalado previamente, examinar qué factores validan el posicionamiento de un 
agente cultural en el campo de la producción cultural en Nuevo León, México. La pregunta de investigación que se intentó responder fue la siguiente: ¿por qué medios se posiciona un individuo como agente cultural, y qué valores le caracterizan como tal, en el contexto artístico en Nuevo León? Las principales fuentes de información utilizadas para la resolución de esta interrogante fueron diversos estudios sobre el funcionamiento de los mundos del arte y la organización del ámbito cultural y la información recopilada a través de entrevistas semiestructuradas individuales con agentes culturales locales que poseen una trayectoria definida. Además, para la selección de éstos, se establecieron dos criterios centrados en el involucramiento activo de estos en la escena artística neoleonés.

\section{Participantes}

Los dos criterios para seleccionar a los entrevistados de la investigación fueron, primeramente, el de las actividades que los participantes potenciales han realizado a lo largo de sus vidas profesionales, ya sea en la creación de obras de arte, promoviendo eventos culturales, administrando instituciones culturales $\mathrm{y} / \mathrm{o}$ educativas, o cumpliendo todas estas actividades de manera simultánea. El segundo criterio fue el de los años de experiencia de cada participante potencial, así como su grado de implicación en el campo local de la producción cultural. La experiencia promedio de cada entrevistado fue de 10 a 20 años; en cuanto al grado de participación, se tomó en cuenta el que éstos se desempeñen de forma ininterrumpida ya sea como artistas, promotores o gestores culturales, o como profesores de arte. Las características sociodemográficas de los participantes comprendían el nivel académico y su edad. Todos los posibles entrevistados debían tener un perfil muy específico que consistía en que posean una licenciatura como nivel mínimo de educación, o bien maestría o doctorado, como máximo nivel educativo. Por el grado académico y la experiencia requerida, la edad de cada posible participante debía ser de 35 años o mayores.

Cabe señalar que ambos criterios de selección se basan parcialmente en los formulados por Peist (2011) en su estudio sobre el reconocimiento en el ámbito de 
las artes; además, los criterios empleados en este trabajo se sustentan de manera general en la propuesta de García-Canclini (2014) para el estudio sociológico del arte, y en particular, en un aspecto metodológico relevante al análisis de la estructura interna del campo artístico, que es el de las relaciones sociales de producción (p. 93), lo que atañe tanto a las prácticas de elaboración de mensajes artísticos (la creación de obras y actos o performances artísticas), así como a las formas en que los artistas interactúan entre sí (como formadores de otros artistas, como gestores de galerías o museos, o como promotores o críticos de otros creadores culturales) y al nivel en el que éstos se posicionan en el campo de producción cultural (p. 94).

\section{Instrumento}

La estrategia de investigación empleada fue la entrevista semiestructurada; este tipo de entrevista se centra principalmente en las percepciones y perspectivas del entrevistado, con el fin de obtener respuestas lo más amplias posibles para obtener información detallada sobre el fenómeno investigado (Bryman, 2012). Las entrevistas estuvieron compuestas por cinco preguntas abiertas, orientadas a examinar las opiniones y percepciones de los participantes acerca de los factores que validan las actividades de los agentes culturales; dichas preguntas se elaboraron siguiendo las recomendaciones señaladas por Corbetta (2007), atendiendo principalmente a la brevedad y sencillez de las interrogantes, a evitar términos ambiguos $\mathrm{y} / \mathrm{o}$ palabras que pudieran suponer algún prejuicio 0 preconcepción respecto a las actividades de los participantes. En la Tabla 1 se expone la guía original de las preguntas empleadas en esta investigación. Durante las entrevistas, se alentó a los participantes de la investigación a agregar todos los comentarios necesarios para así obtener datos complementarios. Para el análisis de la información recabada a través de las entrevistas, se utilizó el método de codificación de patrones para reducir los datos a temas específicos en los que se identificaron ciertos patrones (Álvarez-Gayou, 2012). Los resultados obtenidos se organizaron en tres temas principales que se exponen en la siguiente sección. 
Tabla 1.

Relación de preguntas empleadas en las entrevistas

\section{Preguntas}

1 ¿Qué influencias, movimientos o tendencias caracterizan tu producción artística?

2 ¿Qué habilidades o aptitudes debe poseer todo artista para ser exitoso?

3 ¿Qué medios son los más adecuados para dar a conocer el trabajo de un artista?

4 ¿Qué tan fácil o difícil es integrarse a la escena artística en el estado?

5 ¿De qué forma se valida el trabajo de un artista?

Es pertinente mencionar que hubo algunos problemas para llevar a cabo este estudio. En primer lugar, se convocó a quince agentes culturales en función de su implicación en el ámbito local de la producción cultural y su experiencia como artistas, promotores o docentes. De éstos, sólo once aceptaron ser entrevistados. Los otros cuatro no respondieron a la solicitud de colaborar con esta investigación. El segundo problema fue la delimitación del fenómeno examinado. Debido al número de entrevistados, fue necesario reformular las entrevistas para garantizar que los participantes de la investigación dieron respuestas más detalladas y, en consecuencia, obtener suficiente información para una comprensión profunda de las condiciones y valores por los que un agente cultural se posiciona y acredita dentro del campo de la producción cultural del estado de Nuevo León, México.

El replanteamiento de las preguntas, como se muestra en la Tabla 2, se basó principalmente en corregir las interrogantes que se prestaran a equívocos, como en el caso de la pregunta número 2 , en la que el concepto de éxito puede referirse al comercial o económico, al cultural, al social, etc.; así mismo, se optó por explicitar y aclarar lo mejor posible cada uno de los temas a los que aluden las preguntas, como en el caso de la número 5, que en su primera versión, presentaba de una manera muy vaga e imprecisa la noción de valoración, dando cabida a concepciones subjetivas referentes al artista (si es una persona agradable o desagradable, 
oportunista o talentoso, etc.), mientras que el concepto planteado en este estudio se refiere específicamente a la percepción, positiva o negativa, sobre el quehacer artístico de tal o cual agente cultural.

\section{Tabla 2.}

Relación de preguntas reformuladas empleadas en las entrevistas

\section{Preguntas (reformuladas)}

1 ¿Consideras que adherirse a alguna tendencia, movimiento o escuela artística-estética garantiza el ingreso a la escena artística neoleonesa?

2 ¿Qué habilidades, aptitudes y/o conocimientos requiere un artista para dar a conocer su trabajo?

3 ¿A través de qué canales puede un artista validar o legitimar sus actividades creativas?

4 ¿Qué tan fácil o difícil es integrarse a la escena artística en el estado?

5 ¿Qué factores intervienen en la valoración positiva o negativa del trabajo de un artista? 


\section{Procedimiento}

La presente investigación se planteó describir qué factores y valores acreditan a un creador artístico, promotor cultural o docente de las artes, como un agente cultural en un ámbito local de producción cultural. El análisis general de todos los datos compilados se realizó confrontando las teorías sobre la constitución del mundo del arte y los modelos estructurales de la cultura con la información proporcionada por los entrevistados. El análisis de las percepciones y experiencias de once agentes culturales reconocidos permitió obtener información sobre las formas de posicionamiento y los valores que se deben realizar dentro de un campo local de producción cultural. La investigación se centró en Nuevo León, específicamente en Monterrey y San Pedro Garza García, municipios con una mayor oferta cultural y con una tradición consolidada de liderazgo económico en el estado.

Como se mencionó en la metodología, los criterios de selección de participantes fueron las actividades que realizan en el campo cultural, sea en la creación artística, la promoción cultural o la docencia de las artes, así como su trayectoria profesional. Cabe reiterar que, de los quince agentes culturales convocados para participar en la investigación, sólo once accedieron a ser entrevistados. Es necesario indicar que, de los once participantes, cuatro se desempeñan como artistas a la vez que como profesores, tres son artistas, profesores y promotores, dos son promotores y profesores, y los últimos dos son artistas, galeristas y promotores también. Todos los participantes de la investigación cuentan con una experiencia en el campo de diez a veinte años y cada uno de ellos se desempeña activamente en la producción artística, la promoción cultural y la gestión, la enseñanza de las artes y la investigación artística. 


\section{Resultados}

Los resultados obtenidos a partir del análisis permiten construir una visión general del funcionamiento interno del campo local de producción cultural, con base en las opiniones, el conocimiento y la implicación directa de los agentes culturales entrevistados. Los resultados se clasificaron en los siguientes rubros o temas: a) condiciones que determinan el posicionamiento en el campo local de la producción cultural y que pueden estar relacionados ya sea con el ámbito de la producción o con el de la distribución; b) los instrumentos y factores que validan el trabajo cultural, tales como la crítica especializada, los espacios y eventos institucionalizados, o el éxito en el mercado; y c) las percepciones de los entrevistados sobre la apertura e inclusión del campo local de la producción cultural.

\section{Condiciones de posicionamiento}

De acuerdo a las respuestas brindadas por los participantes de la investigación, hay dos clases principales de circunstancias que definen la inclusión de una persona que está dispuesta a pertenecer a la escena cultural local. En primer lugar, factores tales como los valores estéticos dominantes o la adhesión estilística a cierto movimiento artístico o escuela filosófica. En segundo lugar, las relacionadas con el ámbito de la distribución cultural, que incluye habilidades como la autogestión y autopromoción, y el trabajo colaborativo.

En relación con esto, ocho de los once entrevistados afirmaron que los valores estéticos son los factores más importantes que influyen en el posicionamiento en el campo local de la producción cultural. Los otros tres señalaron que todas las habilidades referidas al ámbito de la distribución son las que posibilitan que un artista se posicione en la escena cultural neoleonés. No obstante, todos los participantes de la investigación complementaron posteriormente sus respuestas argumentando que existe una influencia recíproca entre los valores estéticos que determinan la producción artística y las competencias 
de autogestión y autopromoción, que posibilitan la distribución de las obras artísticas. Desde el punto de vista de los participantes, una elección estilística o los valores expresados en una obra de arte influyen y son influenciados por la capacidad de autogestión del actor cultural o por su pericia en la promoción de una obra o evento cultural particular. La Tabla 3 sintetiza las respuestas de acuerdo a los conceptos prioritarios señalados por los participantes.

Tabla 3.

Relación de respuestas por conceptos predominantes del tema 1

\begin{tabular}{|c|c|c|}
\hline Pregunta & Respuesta (conceptos) & Frecuencia \\
\hline $\begin{array}{l}\text { ¿Consideras que adherirse a alguna } \\
\text { tendencia, movimiento o escuela } \\
\text { artística-estética garantiza el ingreso a }\end{array}$ & $\begin{array}{llr}\text { Adhesión } & \text { a } & \text { ideas, } \\
\text { tendencias } & \text { y } & \text { valores } \\
\text { estéticos } & & \end{array}$ & $8 / 11$ \\
\hline la escena artística neoleonesa? & $\begin{array}{l}\text { Contactos, relaciones con } \\
\text { personajes clave del } \\
\text { campo artístico }\end{array}$ & $3 / 11$ \\
\hline $\begin{array}{l}\text { ¿Qué habilidades, aptitudes y/o } \\
\text { conocimientos requiere un artista para } \\
\text { dar a conocer su trabajo? }\end{array}$ & $\begin{array}{l}\text { Autogestión, } \\
\text { autopromoción y trabajo } \\
\text { colaborativo }\end{array}$ & $11 / 11$ \\
\hline
\end{tabular}

\section{Estrategias de validación del actor cultural}

Según las respuestas de los entrevistados, hay tres medios o canales principales que ratifican las actividades de un agente cultural que aspira: la crítica especializada, participación y presencia en espacios y eventos institucionalizados y el éxito en el mercado artístico y cultural. Cinco de los once participantes declararon que la forma más efectiva de validar el trabajo de un artista, un promotor o gestor cultural o un educador artístico son los ensayos críticos o las reseñas especializadas sobre su trabajo; otros cinco de los entrevistados consideran que lo que valida el trabajo de un agente cultural es su participación en eventos avalados por una 
institución cultural, tales como museos, galerías o centros culturales. Sólo un participante señaló que el éxito comercial era el medio adecuado para "medir" y legitimar el trabajo de un actor cultural. La Tabla 4 presenta la relación de respuestas de los entrevistados.

Es pertinente sintetizar los comentarios adicionales de los participantes de la investigación sobre este tema en específico. Todos los entrevistados indicaron que existen factores o circunstancias externas, así como otros mecanismos internos que intervienen en la organización del campo local de producción cultural, influyendo así tanto en el posicionamiento como en la acreditación de los artistas. Las circunstancias externas se refieren a cuestiones políticas, específicamente a la transición de un régimen a otro, con las consecuencias que ello conlleva: la cancelación de ciertas políticas públicas sobre el desarrollo cultural y la implementación de otras diseñadas por los funcionarios del régimen actual; los recortes al presupuesto público y la escasez de subsidios y apoyos a las artes y la cultura. Otra dinámica interna que también influye en el posicionamiento y la validación de los agentes culturales, según consenso de los entrevistados, son las asimetrías entre los discursos artísticos y las competencias estéticas de las audiencias.

Tabla 4.

Relación de respuestas por conceptos predominantes del tema 2

\begin{tabular}{lllc}
\hline & Respuesta (conceptos) & Frecuencia \\
\hline ¿A través de qué canales puede un & Ensayos críticos, reseñas & $5 / 11$ \\
artista validar o legitimar sus & especializadas & \\
actividades creativas? & $\begin{array}{l}\text { Participación en eventos } \\
\text { institucionales } \\
\end{array}$ & $5 / 11$ \\
& Éxito comercial & $1 / 11$ \\
\hline
\end{tabular}




\section{El campo artístico desde la óptica de sus actores}

En lo que respecta a las percepciones y opiniones de los participantes en torno a la apertura e inclusión de la escena cultural local, es decir, la facilidad o dificultad con la que un artista novel se integra al ámbito artístico regional, siete de los entrevistados opinaron que, en su estado actual, el campo local de la producción cultural es una esfera más democrática e inclusiva. Los otros cuatro participantes de la investigación consideraron que posicionarse y ser acreditado como agente cultural es un proceso muy difícil y depende en parte de circunstancias extrínsecas al ámbito artístico y cultural neoleonés.

Los entrevistados que afirmaron que existe una mayor apertura e inclusión en la escena cultural local también indicaron que el posicionamiento y la acreditación depende de factores tales como el poder elaborar un discurso artístico propio, de la disposición para colaborar con otros artistas, educadores de arte, promotores y gestores culturales, y el poseer habilidades de autopromoción y autogestión. Los participantes que aseguraron que la inclusión y validación en el campo local de la producción cultural es sumamente problemática agregaron que existen también factores externos que obstruyen el posicionamiento y la acreditación de artistas emergentes o nóveles, reiterando circunstancias tales como los recortes y la centralización de los fondos y recursos públicos que usualmente se destinaban al arte y la cultural, la falta de apoyo al desarrollo artístico y cultural y la interacción irregular entre actores culturales y las audiencias (véase Tabla 5). 


\section{Tabla 5.}

Relación de respuestas por conceptos predominantes del tema 3

\begin{tabular}{|c|c|c|}
\hline Pregunta & Respuesta (conceptos) & Frecuencia \\
\hline \multirow[t]{2}{*}{$\begin{array}{l}\text { ¿Qué tan fácil o difícil es } \\
\text { integrarse a la escena } \\
\text { artística en el estado? }\end{array}$} & $\begin{array}{l}\text { Mayor facilidad de inclusión; mayor } \\
\text { democratización de la esfera artística } \\
\text { local }\end{array}$ & $7 / 11$ \\
\hline & $\begin{array}{l}\text { Mayor dificultad de integración a la } \\
\text { esfera artística local }\end{array}$ & $4 / 11$ \\
\hline \multirow[t]{2}{*}{$\begin{array}{l}\text { ¿Qué factores intervienen } \\
\text { en la valoración positiva o } \\
\text { negativa del trabajo de un } \\
\text { artista? }\end{array}$} & $\begin{array}{l}\text { Factores intrínsecos: elaboración de un } \\
\text { discurso artístico propio; disposición } \\
\text { para colaborar con otros artistas, } \\
\text { educadores de arte, promotores y } \\
\text { gestores culturales; habilidades de } \\
\text { autopromoción y autogestión }\end{array}$ & $7 / 11$ \\
\hline & $\begin{array}{l}\text { Factores extrínsecos: recortes y } \\
\text { centralización de fondos y recursos } \\
\text { públicos destinados al arte y la cultural; } \\
\text { falta de apoyo al desarrollo artístico y } \\
\text { cultural; interacción irregular entre } \\
\text { actores culturales y las audiencias }\end{array}$ & $4 / 11$ \\
\hline
\end{tabular}

\section{Discusión}

Los resultados descritos anteriormente permiten inferir qué aptitudes y características ha de poseer un agente cultural para integrarse a la escena cultural específica del estado de Nuevo León, México. La capacidad para cumplir con varias actividades es uno de los atributos deseables de un artista en la actualidad: tiene que ser capaz de crear obras de arte, así como de promover y gestionar sus propias actividades, y también de elaborar un discurso crítico sobre su propio trabajo. En 
otras palabras, el agente cultural debe ser creador, promotor y crítico de su propio trabajo.

Una posible explicación para este fenómeno sería que, usualmente, los galeristas, promotores y críticos son mediadores entre el arte y el público, por lo que pueden influir en la valoración económica de la obra de arte, en los canales de su distribución, así como en su recepción y percepción. Sin embargo, estos intermediarios pertenecen al dominio de la distribución del mundo del arte (Maanen, 2009), lo que significa que no están directamente involucrados en la creación de bienes culturales y artísticos. Así pues, en la búsqueda de la autonomía y la autosuficiencia, así como para acortar la distancia entre el arte y el público, los creadores culturales asumieron las funciones de promoción, gestión y pensamiento crítico.

Los resultados también apuntan a la existencia de tres discursos predominantes sobre el posicionamiento y legitimación de los agentes culturales en el estado. El primero se refiere a su participación en eventos y espacios institucionalizados, el segundo es el reconocimiento académico que pueden lograr a través de sus actividades y el tercero alude a su éxito en el mercado (Stallabras, 2004). Según la investigación, hay una mayor adhesión a la validación institucional y una cohesión mínima a los logros económicos. Sin embargo, los agentes culturales que se adhieren el discurso institucional pertenecen a esos mismos organismos culturales públicos, es decir, ya se encuentran posicionados en el campo de la producción cultural, y son reconocidos en el medio cultural como artistas, promotores o gestores. Es pertinente señalar que todos los entrevistados son conscientes de la asunción de un modelo corporativo por instituciones culturales públicas y privadas (Stallabras, 2004). Este cambio afecta radicalmente las oportunidades de financiamiento del desarrollo artístico (Maanen, 2009) y, en consecuencia, las posibilidades de artistas que aspiran a insertarse dentro del ámbito cultural y ser acreditados por sus pares consolidados.

Además, y debido a la escasez de financiamiento público para las artes y la cultura, los actores culturales noveles deben financiar sus actividades recurriendo 
al apoyo familiar, al patrocinio privado o a trabajos por encargos o eventuales (Maanen, 2009). Además de lo económico, hay aspectos políticos que influyen en la producción y distribución cultural. La libertad artística y cultural, así como los valores que los agentes culturales deben realizar, dependerán de la orientación de las políticas públicas. Además, de acuerdo con los principios de legitimidad propuestos por Bourdieu (2002), hay un proceso que todos los agentes culturales deben completar. En primer lugar, deben ser reconocidos por otros agentes culturales (principio específico de legitimidad), lo que significa que han ingresado y se han posicionado en el campo de la producción cultural. En segundo lugar, deben obtener la aprobación de las autoridades institucionales, tales como museos o universidades (principio institucional de legitimidad), de esta manera, se les acredita como agentes culturales. Por último, si son reconocidos por el público masivo, si llegan a ser "popularizarse", habrán alcanzado la consagración resultado de la elección de los consumidores ordinarios (Bourdieu, 2002).

\section{Conclusiones}

Una de las problemáticas recurrentes en el campo artístico neoleonés es la de la creación de públicos y consumidores, cuestión que ha ocupado, y preocupado, a artistas, promotores, gestores y educadores y cuyas consecuencias más arraigadas en la sociedad neoleonesa abarcan desde la representación negativa del artista como un sujeto destinado a la precariedad y al pluriempleo (Throsby, 2008) hasta la dificultad para profesionalizar las actividades artísticas, o lo que es lo mismo, el incorporar a los creadores al mercado laboral de una manera equitativa y en condiciones de igualdad con respecto a otras profesiones (O'Connor, 2010). Las posibles causas de esta problemática son el hermetismo y la centralización del ámbito artístico, según lo señalado en las respuestas de los participantes de este estudio, así como los recortes frecuentes al presupuesto destinado a la cultura, área que, dentro de los retos nacionales, no se considera prioritaria. 
El propósito de este estudio fue el de proporcionar una visión, aunque general, del funcionamiento de un campo local de producción cultural a través de las relaciones y dinámicas de sus propios actores. A través del análisis de los aspectos y factores que posibilitan la acreditación como agente cultural, puede señalarse que las circunstancias económicas y políticas tienen un impacto directo tanto en la organización de la escena cultural neoleonesa, así como en las interacciones que establecen sus actores. La distinción entre el ámbito de la producción cultural y el contexto de la distribución cultural, sustentada en las premisas de Maanen (2009), permitió comprender por qué un agente cultural a menudo es creador, promotor, gestor y crítico al mismo tiempo. Por otra parte, las tesis de Bourdieu fueron esenciales para el discernimiento de la lógica y los procesos del campo de la producción cultural. Aunque esta investigación se limitó al estado de Nuevo León, su alcance pudiera ampliarse para conocer las condiciones específicas de la escena cultural de otros estados de la república. 


\section{Referencias}

Alexander, J. C. (2000). Sociología cultural. Formas de clasificación en las sociedades complejas. Barcelona: Anthropos.

Álvarez-Gayou, Jurgenson, J. L. (2012). Cómo hacer investigación cualitativa. Fundamentos y metodología. México: Paidós.

Amores, J. E. (2007). Monterrey: una cultura propia. En Ortega Ridaura, Isabel (coord.). Nuevo León en el siglo XX. Del Segundo auge industrial a la crisis de 1982. Monterrey: Fondo Editorial de Nuevo León. 73-107.

Benjamin, W. (2003). La obra de arte en la época de su reproductibilidad técnica. México: Itaca.

Bourdieu, P. (1992). Las reglas del arte. Génesis y estructura del campo literario. Barcelona: Anagrama.

Bourdieu, P. (2002). Campo de poder, campo intelectual. Itinerario de un concepto. Tucumán: Montressor.

Bryman, A. (2012). Social Research Methods. New York: Oxford University Press.

Corbetta, P. (2007). Metodología y técnicas de investigación social. Madrid: McGraw-Hill.

Davies, R. y Sigthorsson, G. (2013). Introducing the Creative Industries. From Theory to Practice. California: Sage Publications.

Dickie, G. (2001). Art and Value. California: Blackwell Publishers Inc.

Florida, R. (2012). The Rise of the Creative Class. New York: Basic Books.

García-Canclini, N. (2014). La producción simbólica. Teoría y método en sociología del arte. México: Siglo XXI.

Guillory, J. (1994). Cultural Capital. The Problem of Literary Canon Formation. Chicago: The University of Chicago Press.

Groys, B. (2014). On the New. New York: Verso.

Hartley, J. et al (2013). Key Concepts in Creative Industries. California: Sage Publications.

Heinich, N. (2002). Sociología del arte. Buenos Aires: Nueva Visión.

Howkins, J. (2013). The Creative Economy. How People Make Money from Ideas. London: Penguin Books.

Luhmann, N. (2005). El arte de la sociedad. México: Herder.

Maanen, H. (2009). How to Study Art Worlds. On the Societal Functioning of Aesthetic Values. Amsterdam: Amsterdam University Press.

Moulin, R. (2012). El mercado del arte. Buenos Aires: la marca editora.

Nivón-Bolán, E. y Villalobos-Audifred, H. (2006). Perfil metropolitano del consumo cultural. Los casos de Guadalajara, Monterrey y el Distrito Federal. En Arizpe, L. (coord.). Retos Culturales de México frente a la globalización, México: Miguel Ángel Porrúa. 535-559.

ISSN: 2007-1833 
O'Brien, D. (2014). Cultural Policy. Management, Value and Modernity in the Creative Industries. New York: Routledge.

O'Connor, J. (2010). The Cultural and Creative Industries: A Literature Review. London: Creative, Culture and Education.

Peist, N. (2011). Éxito y arte moderno. Trayectorias artísticas y proceso de reconocimiento. Madrid: Abada.

PNUD (2014). Informe sobre la economía creativa. México: UNESCO. Recuperado el 7 de septiembre en: http://www.unesco.org/culture/pdf/creative-economy-report-2013-es.pdf.

Rubio-Elosúa, E. (2000). De promotores, instituciones y políticas culturales. En Moyssén, X. (ed). Artes Plásticas de Nuevo León. 100 años de historia, siglo XX. Monterrey: Museo de Monterrey. 155-196.

Stallabras, J. (2004). Art Incorporated. The Story of Contemporary Art. New York: Oxford University Press.

Throsby, D. (2008). Economía y cultura. México: CONACULTA.

Towse, R. (2010). A Textbook of Cultural Economics. New York: Cambridge University Press.

Walliman, N. (2011). Research Methods. The Basics. New York: Routledge. 